\author{
International Journal of Linguistics, Literature and Translation (IJLLT) \\ ISSN: 2617-0299 (Online); ISSN: 2708-0099 (Print) \\ DOI: $10.32996 / \mathrm{ijllt}$ \\ Journal Homepage: www.al-kindipublisher.com/index.php/ijllt
}

IJLLT

\title{
Tunisian Students' Perception and Awareness of Metadiscourse Stances in Reading an Academic text
}

Dr. Maha Dallagi,

Lecturer in Applied Linguistics, Business English Department, Higher Institute of Applied Studies in Humanities of Zaghouan, University of Tunis, Tunisia

Corresponding Author: Dr. Maha Dallagi,,E-mail: maha_dallagi@yahoo.co.uk

\author{
ARTICLE INFORMATION \\ Received: November 03, 2020 \\ Accepted: January 12, 2021 \\ Volume: 4 \\ Issue: 1 \\ DOI: 10.32996/jillt.2021.4.1.4
}

\section{KEYWORDS}

Metadiscourse, Reading, Comprehension, Lexical Invisibility,

Tertiary level

\section{ABSTRACT}

'Lexical Invisiblity' or failures in perceiving metadiscourse signs could impact students' comprehension of text, especially academic ones. In the Tunisian educational system which divides Tertiary level students into broad specialisms, teachers adopt various reading approaches accordingly to students' needs and requirements of their professional careers. This might lead to a focus on some linguistic aspects at the expense of others, and might prevent learners from understanding the conveyed message at its fullest. The current research aims to determine to what extent Tunisian University learners $(\mathrm{N}=12)$ are aware of the presence of stances, while reading an academic text. Echoing Low's (1996) and Hyland's (2003) studies, a Think- Aloud Protocol permitted to identify students' shortage in perceiving interactional items, such as hedges and boosters. Findings indicate that students tend to focus on the overall content and make a total abstraction of the linguistic signs of certainty and uncertainty. The investigation stresses the importance of highlighting the linguistic means that enhance the comprehension of academic texts. As overlooking interactional devices could affect students' accurate understanding of academic texts, EFL instructors are strongly suggested to raise learners' awareness of Metadiscourse items by teaching them explicitly and drawing their attention to them.

\section{Introduction}

EAP learners must not only understand the content of the message conveyed, but also distinguish the communicative function of sentences in academic texts. Yet, the students' needs and curriculum could influence teachers' choice of approaches. Document survey of Tunisian Material used in teaching EFL to English, French, Medicine and Engineering majors, unveiled that Hard science students are more likely to get a vocabulary-oriented instruction. EFL Teachers in Soft sciences seem to be keener on accentuating the discourse-oriented reading approach. Nonetheless, this lack in highlighting the discursive role of items might result in students' misunderstanding of the academic text. Accurate perception of metadiscursive stances, such as hedges and boosters, is necessary in the decoding of writers' message and permit to discern author's stances and to what extent he/she is certain. Awareness of metadiscourse features allows university students to reprocess these means when attempting to express opinion, doubt and certainty. Hyland (2000, p. 109) defines metadiscourse "As the linguistic resources used to organize a discourse or the writer's stance towards either its content or the reader."

A major part of this study focuses on the perception of metadiscourse items or what was referred to by Low (1996) as "Lexical Invisibility". A particular focus will be on hedges and boosters, which are identified by Hyland (2005) as interactional items. Because of their epistemic and affective significance, hedges and boosters are worth investigating in an EAP context and EFL context.

\section{Literature Review}

EAP reading and writing skills require the ability to perceive and express utterances in accordance to their adequate communicative function. These characteristics put into value the significance of metadiscourse items in academic texts. Reading

K C AL-KINDI CENTER R D FOR RESEARCH AN Your gateway to world-class research

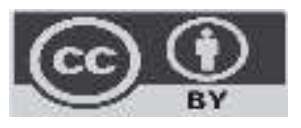

Published by Al-Kindi Center for Research and Development. Copyright (c) the author(s). This open access article is distributed under a Creative Commons Attribution (CC-BY) 4.0 license 
is an important challenging linguistic and thinking process, and EAP students must be able to recognize the sense of all lexical items in order to fulfill all course objectives (Perfetti \& McCutchen, 1987; Grabe, 1991; Lynch \& Hudson, 1991). In second language curricula, EAP courses focus more on the expressions of ideational meanings whereas hedges and boosters are overlooked; consequently, students unnotice these devices once they encounter them in a text (Hyland, 2000).

The objective of this study was to determine to what extent students are aware of metadiscourse items, with a particular focus on Hedges and Boosters. The latter, referred to also as Downtoner and Intensifiers allow academics to express conviction by adopting a degree of vigilance and by conveying an "Appropriate disciplinary persona of modesty and assertiveness" (Hyland, 1996). For Hyland (2000, p. 1), "Might, probably and seem are hedges that signal a tentative assessment of referential information and convey collegial respect for the views; and boosters such as clearly, obviously and of course allow writers to express conviction and to mark their involvement and solidarity with the audience". Lakoff (1972, p. 195) was among the first scholars to be interested in downtoners which he referred to as "hedges". Items such as 'I think', 'perhaps', 'might' or 'maybe' are for Lakoff "words whose job is to make things more or less 'fuzzy". Hyland (1994) explains that academics are crucially concerned with varieties of cognition, and cognition is inevitably 'hedged'. The writer cannot always claim with certainty any given information and must anticipate the reader's reaction to his/ her assertion. Yagiz and Demir (2015, p. 12) describe boosters as "intensity markers that prove the author's stance on a colossal scale by narrowing discursive space." These intensity markers seem to contradict the principle of tentativeness and caution strategies as they demonstrate writers' commitment and confidence in asserting a claim, preventing the reader from the right to comment and negotiate the information (Hyland, 2005).

Dee-Lukas and Larkin (1986) notice that despite the crucial rhetorical and affective importance of these devices in academic writing, hedges and boosters are of little interest in the educational context. Hyland (1994) indicates that most EAP materials insist more on the way referential information is reached, neglecting the epistemic aspect of the text. Carr and Curren (1994) argue that the absence of explicit linguistic instruction negatively impacts students as it prevents them from acquiring fundamental practices in interacting with the reader while writing. If students are unaware of these devices, they are unable to perceive the communicative function existing in an academic text and the author's "social negotiation of knowledge" (Hyland, 1998, p. 3).

Hyland (2000) notices that academics are more interested in acquiring subject specific content knowledge; and might then miss to notice the significant contribution of the interpersonal features which characterize the information. This unawareness or failure in perceiving the devices is defined by Low (1996) as 'Lexical Invisibility Hypothesis'. Low (1996) investigated students' attitudes and reactions to intensifiers using a Likert-Type questionnaire. He was inspired by Gaskell, Wright, and O' Muircheartaigh (1993) who focused on interviewees' reactions to words like 'very, extremely and really'. The study by Gaskell et al. (1993) revealed that unless the intensifiers (very, extremely and really) were part of a close or idiomatic collocation, they were quite invisible to respondents. The investigation permitted to draw three main conclusions:

(i) "For most of the questions, the intensifiers made no difference even when the interviewers were instructed to emphasize the intensifier when reading the question; (ii) the reason was because respondents were not processing them adequately. (iii) Our advice is to avoid using intensifiers in question stem" (Gaskell et al, 1993, p. 502).

Low $(1996$, p. 4) tried to determine if students reacted to the presence of hedges and boosters in questionnaires. He attempted to verify 3 hypotheses related to Gaskell et al's (1993) investigation:

-Hypothesis 1: 'very' and 'extremely' are more or less invisible.

-Hypothesis 2: Other foregrounding words used by questionnaire designers are similarly invisible.

- Hypothesis 3: Backgrounding words will be even less possible and will remain almost totally unnoticed.

In an investigation of nine students, Low (1996) tried to determine the extent to which participants perceived hedges (seem and tend) and boosters (very, extremely, far, full, never, and consistently) while reading a text, and the impact of their perception on comprehension. Results revealed that half the students noticed four boosters (very, extremely, far, full) while 'never, consistently, seem and tend' were invisible. The investigation provided data to confirm hypotheses 2 and 3: hedges are unnoticed or less visible than intensifiers on most occasions. With the exception of 'very' and 'extremely', most boosters were invisible (Low, 1996, p. 30).

Nonetheless, Hypothesis 1 could not be verified because of the little sampling he randomly chose for his research without taking into consideration neither the genre nor the academic disciplines. Unlike Gaskell et al. (1993), Low (1996) was unable to confirm the extent to which 'very' and 'extremely' were lexically invisible to participants. The intensifier 'very', was present in 30 questions and was noticed by at least 5 students out of 9 ; whereas, about half the students noticed the presence and the rhetoric meaning 
of 'extremely' (Low, 1996). Gaskell et al. (1993, p. 502) explained that 'extremely' was often perceived as synonymous to 'very', and its rhetoric meaning was then missed.

Hyland (2000, p. 3) hypothesizes that if students do not perceive hedges and boosters in the educational context and are unaware of their meanings, they may fail in "decoding the writer's intention appropriately and miss the propositional information". Hyland (2000) investigated the extent to which 14 Cantonese students were aware of hedges and boosters and their perception of writer's assessment of certainty, while reading an academic text. Participants belonged to two different educational levels: $1^{\text {st }}$ and $3^{\text {rd }}$ year of a BA program. In a first time he made participants undertake comprehension tasks, supported by an interview data where he aimed to bring out students' awareness of hedges and boosters. Likewise previous investigations (Low, 1996; Gaskell et al, 1993), the objective of Hyland's (2000) study was to determine how awareness of these target devices impacted comprehension of the text. In a $2^{\text {nd }}$ task, participants were investigated through a more explicit questionnaire which intended to identify their limits in comprehending particular linguistic items. The results of the investigation revealed a high rate of erroneous answers and students' lacunae in recovering "writer's assessment of certainty". Hyland (2000) argues that students often overlook the target items and focus more on the propositional elements, which give rise to incorrect responses. Data showed that participants provided correct answers in only 50 cases out of 210 possibilities. In $24 \%$ of the cases students succeeded in noticing the presence of hedges and boosters. Hyland (2000, p. 16) explains that "evidence from the retrospective think aloud protocols suggests that while students were often aware of the semantic importance of these devices they systematically failed to attend to them when asked to evaluate assumptions of the truth value of statements in a comprehensive exercise". If participants succeeded in recognizing the effect of at least one booster; hedges were almost invisible even they were clearly detectable.

The second questionnaire shed light on participants' understanding of the target items. Results showed that students were able to recognize to some extent (50\% of correct answers) the forms that altered or weakened the accompanying proposition. $3^{\text {rd }}$ year students unveiled a better proficiency level and were more aware than their counterparts, especially in differentiating between what counts to be "possibly true" and "probably true". However, Hyland (2000) supports that the distinction between the two devices is challenging and tricky.

In sum, the results of the second task (questionnaire) enhanced the findings of the previous one (the think aloud interviews) which revealed that students "do not attend to those devices that add tentativeness and caution to academic statements" (Hyland, 2000, p. 18).

Hyland's (2000) study is in harmony with Low's (1996) general findings. It holds the view that some linguistic items and epistemic meanings seem to be more invisible that others. Participants perceived to some extent the strengthening effect of intensifiers such as 'definitely', 'clearly', 'show' and 'the fact that', but were unable to notice hedges. All participants failed in perceiving the writer's intention of weakening his commitment. Hyland (2000) observed that 'may' and 'might' were easily recognizable, while the meanings of 'speculate' and 'hypothesize' seemed to be unfamiliar to many informants. Students' failure and lack of saliency to recognize the interpersonal devices used by the writer may simply be due to their miscomprehension of the function and meaning of these linguistics signs (Hyland, 2000). This is suggestive of limited lexical background.

\section{Methodology}

Since metadiscourse is a significant aspect of academic performance, this study is also interested in identifying the importance of hedges and boosters in EAP instruction. The ultimate objective is to observe whether the students notice the presence of hedges and boosters while reading an academic text. Hyland (2000) highlights the necessity of drawing teachers' and students' attention to the significance of hedges and boosters. He maintains that the ability to recognize these devices in academic texts is crucial for the acquisition of rhetorical competence in any discipline.

In order to identify participants' degree of awareness, the fieldworker administered a questionnaire (Appendices B and C) to a limited population $(\mathrm{N}=12)$. The questionnaire was supplemented with Think-Aloud protocols. The selection of participants was based on convenience sampling; three majors of each discipline volunteered to participate in the survey. All participants were $3^{\text {rd }}$ year students, aged between 21 and 22 years, and had studied ESP/ EAP for at least 2 years at the tertiary level. They were from Hard Sciences and from Soft Sciences. 2 Males and 10 Females accepted to take part in the think-aloud protocol. The main objective was to identify if students majoring in different specialties perceived hedges and boosters the same way and with the same frequency level.

The questionnaire was inspired by Hyland's instruments (2000) which were used to identify hedges and boosters through oral protocols; it also intended to determine participants' awareness of these interpersonal devices while reading an academic text. 
The researcher opted for an academic text published in the International Journal of Humanities and Social Science, Vol.2 No.5, March 2012 (Appendix A) ${ }^{2}$. Its sections and content were similar to the text used by Hyland and with a parity of hedges and boosters. The text was accessible to different majors. The text was longer than Hyland's (1770words vs 807 words), with a higher frequency of interpersonal items. Participants had to answer two sets of questions (i.e. Task I and Task II) with different instructions; still both sets were meant to evaluate participants' awareness of hedges and boosters.

Task I (Appendix B) is divided in two sections and consists of questions (19 items). It comprises 15 true/false statements and 4 multiple choice questions. These items checked the comprehension of particular sentences to find out whether the students noticed the effects of the metadiscourse devices. These questions intended to raise students' interest in specific sentences which address the "epistemic conviction of the writer" (Hyland, 2000). In other words, participants were evaluated on their ability to notice the conviction of the truth of what the writer states. In this research, following Low's (1996) an Hyland's (2000) codification, students' identification of the key term and explicit answers are identified as direct evidence (D) of their understanding. Indirect evidence (I) identifies cases where participants resorted to alternative phrasings to express equivalent ideas. The fieldworker used a minus (-) to code all inappropriate answers. Gathered information was classified in a table (Table 2).

Task II (Appendix C) consists of 15 statements. It explicitly instructs the participants to identify, on a three-scale continuum ("completely certain", "fairly sure", or "uncertain"), the degree of certainty in what is being said. Participants were instructed to choose "I do not know" if they did not select one of the tree options. All their answers had to be justified by asking them to point to the expression, phrase, or word that guided them in their answers. Various responses permitted to divide the candidates into 3 groups: those who identified hedges and boosters and understood their exact meaning, those who recognized them but missed their exact significance, and those who failed to identify them. Hyland $(2000$, p. 7$)$ explains that the second task seeks "to find out whether a student's lack of response to the strength of a particular statement could be attributed to an inadequate understanding of the meaning of a device, or a failure to bring this knowledge to consciousness when processing the sentence".

In both tasks, the informants were invited to report their thoughts verbally and justify their answers. They were asked to mention the specific items which motivated their responses. The Think-Aloud protocol (TAP) was of significant help in evaluating students' awareness and understanding of hedges and boosters. The fieldworker was able to access to performance process and not just be limited to the analysis of the product. Frequency of Hedges and boosters in Oral protocols were stored in tables. Data were computed on simple percentage calculation which permitted to determine the perception rate across groups of students.

\section{Analysis and Discussion}

\subsection{Questionnaire 1 of the Introspective Think-Aloud Protocol}

Echoing Low (1996) and Hyland (2000), the same evaluative items have been adopted in the analysis of participants' answers . Students' answers were retrieved through think aloud protocol, as they were invited to share their ideas out loud while they were simultaneously thinking about the answers. This procedure permitted the researcher to have access to the cognitive process. All Think-Aloud protocols lasted between 75 and $80 \mathrm{mn}$.

Tables 1 and 2, below, demonstrate students' $(\mathrm{N}=12)$ identification of hedges and boosters in sentences extracted from an academic text (see Appendices A, B, C). The letter D (DIRECT) represents direct, clear and correct answers, the letter I (INDIRECT) stands for correct but indirect explanation and a hyphen (-) signifies student's failure in identifying the linguistic item. The survey is divided into two questionnaires. Questionnaire 1 intended to investigate students' awareness of 9 hedges and 10 boosters (Table 1) while reading an academic text.

Table1: Hedges and Boosters used in the text and questions

\begin{tabular}{|l|l|}
\hline Target items in text and questions & \\
\hline Hedges (9 items) & $\begin{array}{l}\text { Seemed, appeared, believe, suggest, it is believed (passive form), reflect } \\
\text { relatively, might, assumed, hypothesized }\end{array}$ \\
\hline Boosters (10 items) & $\begin{array}{l}\text { Have repeatedly verified, insist, should be taken, have indicated, have } \\
\text { demonstrated, a strong correlation has been discovered, concluded, have } \\
\text { repeatedly demonstrated }\end{array}$ \\
\hline
\end{tabular}

\footnotetext{
${ }^{2}$ Language Learning Strategies among EFL/ESL Learners: A Review of Literature by Pezhman Zare (Marvdasht Branch, Islamic Azad University Marvdasht, Iran.) http://www.ijhssnet.com/journals/Vol 2 No 5 March 2012/20.pdf
} 
Table 2 reports each participant's answers and shows that students attended to hedges and boosters in only 91 out of 228 possibilities, a ratio of $39.91 \%$.

Table 2: Direct (D) and Indirect (I) evidence of attention by question (Q) and by participant in Task 1

\begin{tabular}{|c|c|c|c|c|c|c|c|c|c|c|c|c|c|}
\hline$Q$ & S1E & S2E & S3E & S4F & S5F & S6F & S7M & S8M & S9M & S10E & S11E & S12E & $\begin{array}{l}\text { Total of } \\
\text { mentions }\end{array}$ \\
\hline 1 & - & - & - & - & - & 1 & I & D & - & - & 1 & $D$ & 5 \\
\hline 2 & - & - & - & I & - & - & - & - & - & - & - & - & 1 \\
\hline 3 & - & - & - & - & - & - & - & - & - & - & I & - & 1 \\
\hline 4 & - & - & - & - & - & - & - & - & - & - & I & - & 1 \\
\hline 5 & - & - & - & - & $\mathrm{D}$ & - & - & - & - & - & $D$ & I & 3 \\
\hline 6 & I & D & - & 1 & $\mathrm{D}$ & - & - & - & 1 & I & $D$ & $D$ & 8 \\
\hline 7 & D & 1 & D & 1 & $\mathrm{D}$ & 1 & 1 & - & 1 & 1 & D & D & 11 \\
\hline 8 & - & - & - & - & - & - & - & I & - & I & D & D & 4 \\
\hline 9 & - & - & - & I & D & - & $\mathrm{D}$ & - & - & D & $\mathrm{D}$ & $\mathrm{D}$ & 6 \\
\hline 10 & - & - & - & - & - & - & D & D & - & 1 & - & D & 4 \\
\hline 11 & - & - & I & - & D & - & $\mathrm{D}$ & $\mathrm{D}$ & - & $\mathrm{D}$ & $\mathrm{D}$ & $\mathrm{D}$ & 6 \\
\hline 12 & - & - & $\mathrm{D}$ & - & - & $\mathrm{D}$ & - & $\mathrm{D}$ & - & - & - & $\mathrm{D}$ & 4 \\
\hline 13 & $D$ & - & $\mathrm{D}$ & - & - & $D$ & - & - & 1 & - & - & I & 5 \\
\hline 14 & $\mathrm{D}$ & $\mathrm{D}$ & $\bar{D}$ & - & - & - & D & D & D & - & I & - & 7 \\
\hline 15 & $D$ & - & - & I & $D$ & - & $\mathrm{D}$ & $\mathrm{D}$ & $\mathrm{D}$ & $D$ & - & $D$ & 8 \\
\hline 16 & - & - & - & - & - & D & - & 1 & - & I & I & $D$ & 5 \\
\hline 17 & I & - & - & - & D & - & - & - & - & 1 & D & D & 4 \\
\hline 18 & - & - & - & - & - & - & - & - & - & $\mathrm{D}$ & $\mathrm{D}$ & - & 2 \\
\hline 19 & 1 & - & $\mathrm{D}$ & - & D & - & - & - & $\mathrm{D}$ & - & D & 1 & 6 \\
\hline & \multicolumn{3}{|c|}{16 mentions } & \multicolumn{3}{|c|}{18 mentions } & \multicolumn{3}{|c|}{21 mentions } & \multicolumn{3}{|c|}{36 mentions } & \\
\hline & \multicolumn{6}{|c|}{ Soft Sciences: 34 mentions } & \multicolumn{6}{|c|}{ Hard Sciences: 57 mentions } & $91 / 228$ \\
\hline$\%$ & \multicolumn{6}{|c|}{ 14.91\% } & \multicolumn{6}{|l|}{$25 \%$} & $39.91 \%$ \\
\hline
\end{tabular}

Science students (S7, S8, S9, S10, S11, S12) revealed a higher awareness of hedges and boosters compared to English and French majors (S1, S2, S3, S4, S5, S6) as there are 57 mentions (25\%) among hard science students and 34 mentions (14.91\%) among soft science students.

The results indicate a clear disparity in the participants' recognition of hedges and boosters through their different responses. Some lexical items (to demonstrate, to insist) were recognized while others (seem, believe, appear) were invisible. As indicated in table 3, below, data from the interview reveal that the participants easily recognized boosters compared to hedges.

Table 3: Frequency of recognition of hedges and boosters

\begin{tabular}{|l|l|l|}
\hline Lexical Items & Sentence & $\begin{array}{l}\text { Frequency of } \\
\text { recognition }\end{array}$ \\
\hline Hedges & & \\
\hline Seemed & Sentence 2 & 1 \\
\hline Appeared & Sentence 3 & 1 \\
\hline
\end{tabular}




\begin{tabular}{|l|l|l|}
\hline They believe & Sentence 4 & 1 \\
\hline Suggest & Sentence 5 & 3 \\
\hline It is believed & Sentence 8 & 4 \\
\hline Reflect relatively & Sentence 9 & 6 \\
\hline might include & Sentence 10 & 4 \\
\hline assumed & Sentence 11 & 6 \\
\hline hypothesized & Sentence 12 & 4 \\
\hline & & Total: $\mathbf{3 0}$ \\
\hline & & \\
\hline Boosters & & \\
\hline have repeatedly verified & Sentence 1 & 5 \\
\hline insist & Sentence 6 & 8 \\
\hline should be taken & Sentence 7 & 11 \\
\hline Clearly shown & Sentence 13 & 5 \\
\hline Have demonstrated & Sentence 14 & 7 \\
\hline A strong correlation & Sentence 15 & 8 \\
\hline concluded & Sentence 16 & 5 \\
\hline Significant/have demonstrated & Sentence 17 & 4 \\
\hline Have demonstrated & Sentence 18 & 2 \\
\hline Have repeatedly demonstrated & Sentence 19 & 6 \\
\hline & & Total: $\mathbf{6 1}$ \\
\hline
\end{tabular}

Informants noticed intensifiers 61 times unlike hedges that were identified 30 times.

Concerning intensifiers, participants easily pointed to all verbal phrases with the verb "to demonstrate" in 4 sentences (sentences $14,17,18$ and 19) and the verbal phrase "should be taken" was pointed to by 11 participants.

Other hedges such as "seem" "appear" and "believe" were almost invisible in the text as learners seemed not to pay attention to them. These were recognized only once by two different participants Nonetheless, the verbal phrase "it is believed" was identified by three engineering students. Four science participants recognized expressions and items with the modal "might". Four science majors (S7, S10, S11, and S12) and two French students (S4 and S5) recognized the hedging difference between the adverbs "relatively" and "definitely" and the boosting of the verbs "assume" and "conclude". None of the English majors identified the latter items.

Data of questionnaire 1 reveals that Tunisian participants are unaware of hedges and boosters when reading academic texts. Language majors appear to have the lowest ratio with 34 mentions compared to science majors who recognized hedges and boosters in 57 cases. This discrepancy is probably closely related to different reasons such as students' reading strategies, academic materials in use and the importance given by EFL instructors to interactional metadiscourse items. A Document Survey and Semi-structured interview with EFL teachers permitted to reveal some of the reasons behind learners' unawareness of metadiscourse items.

\subsection{Questionnaire 2 of the Introspective Think-Aloud Protocol}

In a $2^{\text {nd }}$ questionnaire, participants were asked to identify ( 6 hedges) and (9 boosters). Some of these lexical items, such as the verbs "believe", "seem" and "to demonstrate" were used twice in the sentences, purposefully, to see if students were able to identify the verb even if it was conjugated in another tense, or presented in the passive form.

In questionnaire 2 (Appendix C), the instruction for the task was more precise, as students were required to read 15 sentences extracted from the text and to identify the writer's degree of certainty. The researcher asked participants to sort out the degree of certainty and identify any words or phrases that would justify their responses. Table 4 describes the different hedges and boosters investigated in questionnaire 2 .

Table 4: Hedges and Boosters investigated trough Questionnaire 2

\begin{tabular}{|l|l|}
\hline Target items in the questionnaire & \\
\hline Hedges (6 hedges) & $\begin{array}{l}\text { Seemed (3), suggest (5), can be (7), might(9), possibly(10), } \\
\text { seems(11), }\end{array}$ \\
\hline Booster (9 boosters) & Have repeatedly verified (1), concluded (2), Observations and \\
\hline
\end{tabular}


research studies led...(4), it has been consistently reported(6),

argues/could (8), the results of previous research studies have

demonstrated (12), studies have shown/have demonstrated(13),

strengthen the fact(14), clearly highlighted (15).

The researcher used $(+)$ to refer to correct answers and all the cases students succeeded in identifying the correct linguistic device and degree of certainty. A minus (-) indicates all erroneous identifications of the degree of certainty. The letter $N(N=$ nuances) refers to the situation in which the participant recognized the correct lexical item but failed to identify the degree of certainty. In other words, (N) refers to all cases, the participant pointed to the correct downtoner or booster but misunderstood its exact meaning and hesitated between "certain" and "fairly sure", "fairly sure" and "uncertain", and "certain" and "uncertain" (Table 5).

As indicated in Table5, below, the participants correctly identified 93 hedges and boosters out of 180 possibilities (51.66\%) and failed in 26 cases (14.44\%).

Table 5: Recognition of hedges and boosters in questionnaire 2 per specialty

\begin{tabular}{|l|l|l|l|}
\hline & \multicolumn{1}{|c|}{+} & \multicolumn{1}{c|}{$\mathbf{~ N}$} \\
\hline English & 21 & 9 & 15 \\
\hline French & 22 & 8 & 15 \\
\hline Medicine & 23 & 7 & 15 \\
\hline Engineering & 27 & 2 & 16 \\
\hline Total & $\mathbf{9 3 \quad} \begin{array}{r}\text { (Soft science= 43 } \\
\text { Hard science= 50) }\end{array}$ & $\begin{array}{r}\mathbf{2 6} \text { (Soft Science= 17 } \\
\text { Hard Science= 9) }\end{array}$ & $\begin{array}{r}\mathbf{6 1} \text { (Soft science = 30 } \\
\text { Hard Science= 31) }\end{array}$ \\
\hline
\end{tabular}

+ : Correct identification of hedges and boosters

-: Failure in identifying hedges and boosters

$\mathrm{N}$ : Nuances, the participant recognized hedges and boosters but failed in identifying the degree of certainty

However, they were able to recognize the linguistic items but could not identify the exact degree of certainty in 61 cases (33.88\%). Hard Science students identified 50 items compared to Language majors who noticed 43 linguistic devices. Languages majors failed to recognized these in 17 cases whereas hard science students failed 9 times. However, findings show an exact parity in cases of Nuances, between language majors Hard science (Cases of Nuances=31).

Participants' answers to questionnaire 2 reveal that participants had less difficulty in identifying metadiscourse items (51.66\%) than in questionnaire 1 (39.91\%).

In the soft science group, there is no distinct difference between English and French students. English majors recognized hedges and boosters in 21 cases whereas French majors identified 22 cases. French students failed to identify 8 linguistic items as English majors did in 9 cases. Language majors appear to have the same difficulty with the same lexical items. English and French majors missed the degree of certainty when the writer referred to studies and researchers in "Teachers and researchers concluded" (sentence 2), "Observations and research studies" (sentence 4) and "These findings strengthen the fact" (sentence 14).

Though, Science majors identified more devices (50 cases) than language majors, and failed in fewer cases (9 times), Table 34 indicates no difference between Medicine, English and French majors' responses. Engineering majors recognized the highest number of hedges and boosters, and they failed only in two cases. Yet, all participants seemed to be mistaken on the degree of certainty of some terms, hence the exact number of Nuances.

As participants gave different responses in the $2^{\text {nd }}$ questionnaire, it is worth investigating the different lexical items that were easily recognized and the ones that were invisible to the participants. Table 6 summarizes the identification frequency of hedges and boosters. 
Table 6: Frequency of Hedges and Boosters in Questionnaire 2 of the Think-Aloud Protocol

\begin{tabular}{|l|l|l|l|}
\hline \multicolumn{1}{|c|}{ Realisations of Hedges and Boosters } & \multicolumn{2}{|c|}{ Frequency } \\
\hline Hedges & + & - & N \\
\hline Sentence 3: It was realized that learners seemed to be successful... & 2 & 8 & 2 \\
\hline $\begin{array}{l}\text { Sentence 5: ...while the results of studies.... are not based on empirical } \\
\text { findings...they suggest... }\end{array}$ & 8 & 2 & 2 \\
\hline $\begin{array}{l}\text { Sentence 7: .... with this knowledge and awareness....language learning } \\
\text { can be }\end{array}$ & 7 & 2 & 3 \\
\hline Sentence 9: For that reason it might not be appropriate... & 10 & 0 & 2 \\
\hline $\begin{array}{l}\text { Sentence 10: But studying language learning strategies possibly will } \\
\text { help... }\end{array}$ & 8 & 0 & 4 \\
\hline Sentence 11:By referring to the literature it seems that ... & 5 & 2 & 5 \\
\hline TOTAL & $\mathbf{4 0}$ & $\mathbf{1 4}$ & $\mathbf{1 8}$ \\
\hline Boosters & + & - & $\mathbf{N}$ \\
\hline Sentence 1: Research findings...have repeatedly verified & 5 & 2 & 5 \\
\hline Sentence 2: Teachers and researchers concluded & 7 & 1 & 4 \\
\hline Sentence 4: Observations and research studies led & 5 & 3 & 4 \\
\hline Sentence 6: It has been consistently reported & 4 & 0 & 8 \\
\hline $\begin{array}{l}\text { Sentence 8: Rubin (1975) argues that once identified these strategies } \\
\text { could be offered ... }\end{array}$ & 0 & 2 & 10 \\
\hline $\begin{array}{l}\text { Sentence 12: The results of previous research studies have } \\
\text { demonstrated }\end{array}$ & 10 & 1 & 1 \\
\hline Sentence 13: Studies have shown/demonstrated & 8 & 0 & 4 \\
\hline Sentence 14: Strengthen the fact & 5 & 2 & 5 \\
\hline Sentence 15: Clearly highlighted & 9 & 1 & 2 \\
\hline TOTAL & $\mathbf{5 3}$ & $\mathbf{1 2}$ & $\mathbf{4 3}$ \\
\hline
\end{tabular}

Oral-protocols and participants' attitudes revealed that they had less difficulty in identifying some metadiscourse items while others seemed to be invisible to them.

In the Hedges category, Table 6 indicates that learners were able to identify some hedges more than others, such as the expressions: "... language learning "can be", "might not be appropriate...", "strategies possibly will help...", "Results of studies....are not based on empirical findings...they suggest..." Two Medicine majors (MM and NF) easily identified these sentences as indicators of moderate certainty as they easily pointed to the modals "could" and "might", and the adverb "possibly". In sentence (5), 10 students were attentive to the expression "are not based on empirical findings" which expresses uncertainty while the hedging verb "suggest" was invisible to them.

Some hedging items as the verb "argue" and the expression "have consistently reported" confused participants who failed in determining writer's precise degree of conviction while uttering the sentence. Ten participants made out the verb "argue" but could not classify it as an indicator of the writer's moderate conviction. Six students considered that the linguistic item is an indicator of the writer's complete conviction, while four participants misunderstood its communicative function and categorized it as marker of the writers' uncertainty. Participants S6 and S12 claimed that the writer used: "the verb 'to argue' because he is completely sure". Student 7 argued that the writer was completely certain because: "he seems to approve what she (Rubin) said", while student 8 claimed that "as far as the writer refers to Rubin's study, "it is proved and that's why he is completely certain".

In the Boosters category, participants straightforwardly pointed out some items more than others such as the expressions "studies have demonstrated" (10 times) and "clearly highlighted" (9 times). All the students except three Language majors (S1, S2, S4) recognized the verbal phrase "clearly highlighted" as marker of the writer's absolute conviction. S1 considered that the expression is not strong enough and that the writer was fairly sure, while S2 and S4 pointed to it but missed its meaning. The latter considered that "He is reporting ideas but he is not sure". S3 (English major) affirmed: "The writer is completely certain because of the usage of 'clearly highlighted' and the strength of the expression." Whereas, S11 (Engineering major) perceived that the use of the two items 'clearly' and 'highlighted' confirm the writer's absolute certainty. The emphasizing expression "to strengthen the fact" was recognized by 10 participants while only five participants succeeded in identifying its exact degree of 
certainty. The expression "strengthen the fact" was recognized but misunderstood by most Language majors, except participant 6 (French student) who succeeded in identifying its exact degree of certainty. Participants 4 and 9 were unable to identify the writer's degree of conviction though it was very clear to most scientific majors. Student 11 justified the claim: "he is sure, completely certain because he uses the present and the verb "strengthen" (the present is used for general truths)."

Frequency and disparity of answers, as well as recognition and awareness of metadiscourse were not characteristics of participants' academic specialties. Nevertheless, all students, regardless of their field of specialization, succeeded in identifying the metadiscourse function of the items such as "can be" (passive form), "might", "possibly" and "demonstrate"; while the verbs "seem" and "argue" were rather invisible to them.

\subsection{Perception of Hedges and Boosters in Reading: Lexical Invisibility Hypothesis}

The description of the data and results show that Tunisian learners have significant difficulties in identifying hedges and boosters while reading an academic text as these are almost invisible in their reading process. In other words, learners do not realize the importance of the discursive role these devices play and their impact on the audience. These results are compatible with Hyland's (2000) and Low (1996). Since in the Tunisian context learners have been trained to find if information of the text is True or False, they tend to rush to figure it out. Students often make abstraction of the structure and especially linguistic terms that may change the meaning and importance of the utterance. This fact is confirmed by the document survey of the various taching materials used to instruct Tunisian EFL students majoring in the specialisms mentioned in this survey. .

While conducting the TAP, almost all participants displayed the same reading behaviour and strategies. Most students read the sentences in the questionnaire and justified their answers (True or False) by looking for the equivalent sentence in the text, missing metadiscourse devices. Students did not do much thinking, they answered the questions by looking for places in the text where the answer was likely to be, as they skimmed and scanned. They were mainly guided by the strategy of identifying specific words that could lead to the correct response. Consequently, all the other items and details were invisible. This strategy characterizes the profile of different students who read the sentence as separate fragments and not an entity which was holding a meaning in the text.

A few participants (4 out of 12), and more precisely science majors (3 out 12), expressed their perplexity while answering. They "felt" that there was a difference between the sentences proposed in the questionnaire and the content of the text, despite their inability to identify it. This explains why they hesitated in giving a definite answer. The few (4 out of 12) students, who hesitated, re-read cautiously the sentence looking for the difference. They succeeded in identifying hedges or intensifiers once they were aware of the function of the modal. The other candidates ( 8 out of 12) did not pay attention to hedging and boosting. They misunderstood the sentences while looking for details related to content, which is the main reason for their erroneous answers. Most participants limited themselves to the surface structure and meaning of the text, and did not "dig deeper". Students did not try to read between the lines and between "the words". Consequently, they missed a lot as far as the meaning of the text at hand.

This is confirmed by the students' responses to questions 14 and 15 (Task 1). Students did not spend time answering and thinking, as the questions were opposite to what was claimed in the text. They just re-read the sentence to demonstrate that the questions were erroneous. This validates the fact that students are trained to skim and scan. They are instructed to look for clear details that permit to justify right answers and to correct the erroneous ones, at the level of content, not discourse. This is confirmed by the Document survey which demonstrates an absence of focus on discourse, and more precisely hedges and boosters. Additionally, some participants, mainly Language majors, tended to read the sentences as a separate fragment and not as whole entity which holds a meaning. Also, they did not follow the instructions that facilitate the completion of the task. They did not hesitate to look for the answer in previous or following lines as far as it dealt with the same content. This confirms students' "obsession" with information. They often ignore instructions provided by teachers which are meant to save time or guide students to the correct answer.

In Questionnaire 2, participants were required to read the sentences extracted from the text and to explain if the writer was "Completely Certain", "Fairly Sure" or "Uncertain". Surprisingly, students who were unable to identify hedges and boosters in the first task easily recognized the linguistic items in the second task. This fact might be related to the formulation of the task. While in the $1^{\text {st }}$ questionnaire students were asked to find if sentences were True or False, in the second questionnaire the instruction was more precise. Participants had to focus on specific details and more precisely those that indicated the degree of certainty. Items and expressions that were invisible in questionnaire 1 were perceptible and meaningful in questionnaire 2 . The second questionnaire raised students' awareness of hedging and intensifying expressions. Some participants, more precisely, 
proficient learners were aware of the exact meaning of hedges and boosters as they reported in the protocol. They justified their choice by referring to the different functions of probability and possibility expressed by modals such as "may", "might", "could", etc. They realized then that the verb "seem" was not an expression of certainty and that the verb "demonstrate" meant to intensify the claim.

Though some devices were clearly understood, other metadiscourse features were more confusing; hence, the number of nuances $(\mathrm{N}=61)$. The participants recognized the correct linguistic items but missed their exact linguistic communicative function, which led to erroneous answers. They failed to identify the exact degree of certainty. They got confused in distinguishing if the writer was completely certain, fairly sure or uncertain.

However, it is worth mentioning that the informants who had not difficulties in identifying some hedges and boosters missed the hedging function of passive sentences (can be + past participle). Nevertheless, most participants saw the necessity to refer to the text. Lexical invisibility hypothesis of hedges and boosters is closely related to reading strategies. If students do not adopt adequate reading strategies, and skim instructions and important parts of the texts they will miss the main ideas, which will lead to miscomprehension. In the context of this research, learners might have adopted the wrong reading strategies and did not read carefully the instructions provided for them in the reading task. This was clear from their behaviour. Students displayed different attitudes:

-Those who overlooked the text and did not pay attention to details that may hold crucial information about the writer's position and degree of certainty, and how far he/ she wants to involve the audience in his argumentation.

- Those who paid attention to any details and considered the sentence as an addition of different fragments, which may have affected their understanding of sentence and led them into confusion, as they missed the correct meaning of the sentence.

- Those who focused on only one part of the sentence and got satisfied with one information to answer the question, neglecting the rest of the sentence. (They did not read the sentence or the text entirely but rather looked for its gist). Consequently, they provided an incomplete answer if not an erroneous one. Some Students displayed a "lazy" behaviour while reading and did not read "intelligently" or, in other words, they did not read between the lines to exploit the meaning of the different metadiscourse features. They were rather obsessed by reporting faithfully the content.

Document survey of the different materials reveals that students do not deal with academic texts, and no room is given to the teaching of metadiscourse features. This finding confirms the low perception rate of hedges and boosters, especially by Language majors. Science majors were slightly more accurate in their identification of metadiscourse features. It is possible that Science students' interest in details is more elaborate and developed than Humanities students. This could explain participants' diverse Reading behaviour.

Many variables, such as teaching instruction, choice of texts, and proficiency could have an impact on students' awareness of hedges and boosters. This last remark is confirmed by Hyland (2000) and Wingate (2012) who have demonstrated a positive correlation between proficiency and perception of metadiscourse by post graduate students when reading an academic text. They also maintain that teachers' instruction is a main reason behind students' lack of awareness of metadiscourse features.

The results of the current study are in line with Hyland's (2000) and Low's (1996) findings. With the exception of modals such as "could", "might", and "may"; as well as common intensifying expressions such as "demonstrate", "always", and "in fact", hedges and boosters are almost invisible to students.

Yet, it is worth mentioning that high rate of Nuances can be due to a point of view issue that may lead to questioning Hyland's perception of Hedges and Boosters. Tunisian participants recognized the correct linguistic item but failed in identifying the writer's exact degree of certainty. While Hyland (1998) consider that expression such as "research has repeatedly verified" are indicators of writers' certainty, some proficient candidates $(\mathrm{N}=5)$, perceived it as an indicator of the writer's uncertainty because he resorts to "research" to strengthen his claim.

Participants' responses demonstrate that lexical invisibility affects comprehension of the academic text and reading proficiency level. Only proficient students focused on these devices and attempted to decode the writers' tentative and assertive ways. Nonetheless, the critical thinking of the latter could question the discursive meaning of some metadiscourse devices. 


\section{Conclusion}

The purpose of this study was to determine the extent to which university students perceived metadiscourse items while reading an academic text. 12 students participated in the survey, and data was gathered through a think aloud protocol. Participants had to answer two questionnaires with different objectives. The results of the investigation revealed a significant lexical invisibility of hedges and boosters in students' reading process. The informants focused mainly on sentence structure and details that permitted to comprehend the texts. Consequently, the deficiency of recognizing metadiscourse items impacted their reading proficiency level, and led to an incomplete comprehension of the texts. In other words, the participants were unable to decode the writers' exact message. Low's (1996) lexical invisibility hypothesis is confirmed. Despite the low ratio, students recognized more boosters than hedges. They easily pointed out verbs "demonstrate", "may" and "must", while the verbs "seem", "appear", "assume" and passive forms were not noticed.

It is difficult to claim if recognition and awareness of some specific metadiscourse items is related to a particular student group. As the inconsistency of answers did not reveal any relationship with major, awareness of hedges and boosters might be related to the proficiency level of the different candidates. The main limitation to this study is the small number of participants, and further research with a larger population would probably lead to different results.

The main contribution of this study is related to the evaluation of Tunisian students' awareness of metadisourse items, and more precisely hedges and boosters. The present study highlights the importance of downtoners and intensifiers, and strengthens the idea that students' awareness of the different interpersonal items is necessary to comprehend an academic text. Correspondingly, a good command of these linguistic items reveals students' consciousness of the discursive rules and the necessity of being cautious and attentive to the audience while making claims and producing assertions. Further research might explore students' use of metadiscourse items after explicit instruction.

\section{References}

[1] Carr, T. \& Curren, T. (1994). Cognitive factors in learning about structured sequences: applications to syntax. Studies in Second Language Acquisition, 16, 205-230.

[2] Dee-Lukas, D. \& Larkin, J. (1986). Novice strategies for processing scientific texts. Discourse Processes, 9, 329-354.

[3] Gaskell, G., Wright, D. \&O' Muircheartaigh, C. (1993). Reliability of surveys. The Psychologist, 6/11, 500-3.

[4] Grabe, W. (1991). Current Developments in second language reading research. TESOL Quarterly, 25, 375-406.

[5] Hyland, K. (1994). Hedging in academic writing and EAP textbooks. English for Specific

[6] Purposes, 13(3), 239-256.

[7] Hyland, K. (1996a). Talking to the academy: Forms of hedging in science research articles. Written Communication, 13(2), $251-281$.

[8] Hyland, K. (1998b). Boosting, hedging and the negotiation of academic knowledge. TEXT, 18(3), 349-382.

[9] Hyland, K. (2000). Hedges, Boosters and lexical invisibility: noticing modifiers in academic texts. Language Awareness, 9(4), $179-197$.

[10] Hyland, K. (2003). Second Language Writing. Cambridge University Press.

[11] Hyland, K. (2005). Metadiscourse. London. Continuum.

[12] Lakoff, G. (1972). Hedges: A study in meaning criteria and the logic of fuzzy concepts. Chicago Linguistic Society Papers, (8), $183-228$.

[13] Low, G. (1996). Intensifiers and hedges in Questionnaire items and the lexical invisibility hypothesis. Applied Linguistics. 17 (1): 1-37.

[14] Lynch, B. \& Hudson, T. (1991). EST Reading. In M. Celce-Muria (ed) Teaching English as a second or foreign language (2 ${ }^{\text {nd }}$ ed). pp $216-232$. New York: Newsbury House.

[15] Perfetti, C. A., \& McCutchen, D. (1987). Schooled language competence: Linguistic abilities in reading and writing. Advances in applied psycholinguistics, 2, 105-141. https://files.eric.ed.gov/fulltext/ED278962.pdf

[16] Yagiz, O., \& Demir, C. (2015). A comparative study of boosting in academic texts: A contrastive rhetoric. International Journal of English Linguistics, 5(4), 12. 


\section{Appendix A}

Text adapted from:

\section{Language Learning Strategies among EFL/ESL Learners: A Review of Literature}

Zare, P. (2012). Language learning strategies among EFL/ESL learners: A review of literature. International Journal of Humanities and Social Science, 2(5), 162-169.

Line \#

\section{Introduction}

Research findings in the area of second language acquisition have repeatedly verified the significant role that learners can play in the process of language learning. The research has also gone through deliberate changes from teachers and teaching methods towards learners and learning techniques to show its correspondence with these fundamental moves during the past decade (Chamot, 2005; Lee, 2003; Reiss, 1985). While learners received more attention and a more prominent place in research studies on second language learning, so did the engaged strategies and techniques they employed to learn the language and overcome its barriers. From among these techniques, language learning strategies have received a particular attention since the late 1970s (Zare, 2010; Brown, 2007; Hong-Nam and Leavell, 2007; Baker and Boonkit, 2004; Oxford, 2003).

\section{The Good Language Learner}

Many of the initial studies on language learning strategies were aimed at defining the "Good" language learner. As the knowledge of second language acquisition increased during the 1970s, teachers and researchers concluded that no single method of language teaching and research findings would mark the start of universal success in teaching a second language (Brown, 2007). It was realized that certain learners seemed to be successful regardless of methods or teaching techniques. "Certain people appeared to be endowed with abilities to succeed; others lacked those abilities" (p.132). Observations and research studies led researchers (Rubin, 1975; Stern, 1975; Rubin and Thompson, 1994) to describe "good" language learners in terms of personal characteristics, styles, and strategies. They believe that good language learners:

1. Find their own way, taking responsibility for their own learning,

2. Organize information about language,

3. Are creative, and try to feel the language by experimenting its grammar and words,

5. Learn to live with uncertainty by not getting confused and by continuing to talk or listen without understanding every word,

6. Use memory strategies to bring back what has been learned,

7. Make errors work for them and not against them,

8. Use linguistic knowledge, including knowledge of the first language, in learning a second language.

While the results of the studies on defining the good language learner are not based on empirical findings, they suggest characteristics of good language learners as students who are actively involved in language learning and are able to solve problems regarding their own learning. These studies provide a basis for our understanding of what good language learners do to acquire the target language. Once the strategies of successful language learners are identified, these strategies can be taught to less successful learners. It has been consistently reported (Wenden and Rubin, 1987) that all language learners report or have been observed using some type of strategies in learning a foreign or second language. However, they insist that successful language learners have reported to use wider range of learning strategies.

On the contrary, the methods and criteria of determining a good language learner is unclear and under question. Although it is easy to classify a language learner as a good one, if she/he has developed the four basic skills and can use them successfully, it remains difficult to determine whether someone who has only learned one or two of these skills is also a good language learner. Speed of acquisition, learner's previous exposure to English, learner's goal, and student's level of proficiency should be taken into account in determining the good language learner (Sewell, 2003).

However, being aware of the characteristics, techniques, and strategies of a good language learner facilitate students' language learning and help them enhance learning efficiency. In addition, with this knowledge and awareness, students' language learning can be improved through learner training by their instructors.

\section{Classifications of Language Learning Strategies}

Research on language learning strategies started at the 1970s. For the most part, the progress in cognitive psychology had a great effect on the research studies on language learning strategies (Williams and Burden, 1997). In most of the research studies done on language learning strategies, identifying what good learners do to learn a second or foreign language has been the main issue. In 1971 Rubin conducted a study in which the main focus was on the strategies of successful language learners. In her study she argues that, once identified, such strategies could be offered to less successful learners. Rubin (1975) classifies learning strategies according to processes which contribute either directly or indirectly to language learning. It is believed (Wenden, 1986) that reading and discussing the strategies of good language learners is a constructive preliminary activity which can help students to get aware of the concept of learner's strategies. 
described by the researchers. Consequently, these strategies have been classified by many professional experts in the area of language learning (Oxford, 1990; Bialystok, 1981; O'Malley, et al. 1985; Willing, 1988; Stern, 1992; Ellis, 1994). This progress not only helped categorize strategies and link them to a variety of cognitive processing phases during language learning, but also assisted in creating instructional frameworks.

Nonetheless, most of these attempts to categorize language learning strategies reflect relatively the same categorizations of language learning strategies without any fundamental changes. They developed their own taxonomies of strategies according to their research findings by applying different methods of data collection. For that reason, it might not be appropriate to compare them and assess their influence on teaching and learning process. But, studying them possibly will help both language teachers and language learners to understand language learning strategies and different methods which are involved in strategy use.

By referring to the literature, it seems that the most inclusive taxonomy of language learning strategies is provided by Oxford's (1990).

\section{Factors Influencing the Choice of Learning Strategies}

The results of previous research studies have demonstrated that many factors affect the choice learning strategies. Those factors might include degree of awareness, age, sex, nationality, learning style, personality traits, motivation, learning context, and language proficiency (Zare\& Nooreen, 2011; Khamkhien, 2010; Rahimi, et al. 2008; Chamot, 2004; Griffiths, 2003; HongNam and Leavell, 2007; Green and Oxford, 1995; Ehrman and Oxford, 1989).

Studies have shown significant gender differences between males and female language learners in which females have demonstrated to use more and wider range of strategies than males (Zare, 2010; Lee, 2003; Green, and Oxford, 1995; Ehrman and Oxford, 1989). Ehrman and Oxford (1989) assume that women might prefer social communication strategies more than men. However, it is hypothesized that after strategy training, men and women will both show strategy strengths. Moreover, many research studies have explored the relationship between learning strategies and learners' proficiency in which the findings have indicated that more proficient language learners use a greater variety and often a greater number of learning strategies (Rahimi et at., 2008; Griffiths, 2003; Lee, 2003; Anderson, 2005; Bruen, 2001; Green and Oxford, 1995; O'Malley and Chamot, 1990; Ehrman, and Oxford, 1989). Motivation is another influential variable which has been widely examined with respect to its relationship with learning strategies. Findings have demonstrated that learners with high motivation use a significantly greater range of learning strategies than less motivated students (Oxford, 1990; Mclntyre and Noels, 1996; Oxford and Nyikos, 1989).

Moreover, learning styles of language learners play a crucial role in choice of language learning strategies. It has been argued that learning styles and learning strategies of an individual learner can work cooperatively with a given instructional methodology (Oxford, 2003). If a harmony exists between these factors, the learner will perform well, feel confident, and experience low anxiety (Oxford, 2003). Studies in the area have shown that an individual's learning style preferences influence the type of learning strategies that they use (Rahimi et al., 2008; Chamot, 2004; Ehrman and Oxford, 1989). For instance, extroverts have demonstrated strong preference for social strategies, while introverts use metacognitive strategies more frequently (Ehrman and Oxford, 1989). Learners who favor group study are shown to use social and interactive strategies, such as working with peers or requesting clarification (Rossi-Le, 1995).

The findings of research studies in the area of language learning strategies provide a greater understanding of strategy use among EFL/ESL learners and support language instructors and curriculum developers to improve their approaches toward teaching and learning goals. These findings also strengthen the fact that strategy use is a complex phenomenon that interacts with a number of variables. These variables have influences on the use of overall strategies, strategy categories, and individual strategies in different ways. So, to obtain a clear idea of learners' patterns of strategy use, it is important to take all these aspects into consideration (Rahimi et al., 2008).

\section{Language Learning Strategies and Language Learning Achievement}

The findings in the area of language learning strategies have repeatedly demonstrated that the use of language learning strategies leads to better proficiency or achievement in mastering the target language (Lee, 2003; O'Malley and Chamot, 1990; Rahimi et at., 2008; Griffiths, 2003; Hong, 2006; Oxford, 1993). O'Malley et al., (1985) clearly highlighted the importance of learning strategies by defining them as "any set of operations or steps used by a learner that will facilitate the acquisition, storage, retrieval or use of information" (p.23). In a study (O'Malley et al., 1985), it has been found that successful language learners have reported to use more and wider range of learning strategies than less-successful students. The same conclusion has been reached in another study (Green and Oxford, 1995) in which language learning strategies of all kinds were used more frequently by more proficient students. In a different study (Griffiths, 2003), a strong positive correlation between learning strategy use and language proficiency has been discovered. The findings revealed that advanced language learners have reported to employ learning strategies more frequently than elementary students.

In this regard, language instructors should take their students learning strategies into considerations and try to recognize and 
identify students' learning strategies in order to support less successful student to achieve success and master the target language. Teachers have the possibility to identify these strategies through observations, language diaries, questionnaires, interviews and so on. By doing so, teachers will be able to assist language learners to recognize and appreciate the power of language learning strategies in the process of second or foreign language learning. Through learning strategies, teachers can also help the students to maintain their motivation, autonomy, and confidence and keep on going and try to accomplish the goal of learning the target language.

110

\section{Appendix B}

A- Mark these statements on the reading text as either True or False:

1-Research findings in the area of second language acquisition confirmed that learners play an important role in the language learning process. (Introduction, L 2-3)

115

2- Some learners are more successful regardless of methods or teaching techniques (the Good Language Learner, L 14).

3-It was confirmed that certain people are gifted with the abilities to succeed. (The Good Language Learner, L 15)

4- Rubin (1975) and other scholars hypothesized that good language learners organize information about language. (The Good Language Learner, L 16-20)

5- Studies on defining the good language learner definitely characterized him as an actively involved student. (The Good Language Learners, L 29-30)

6- Wenden and Rubin (1987) are among those who are certain that successful language learners have reported to use wider range of strategies. (The Good Language Learner, L 34-35)

7- Sewell (2003) claims that it is necessary to take into consideration the speed of acquisition, learner's previous exposure to English and student's level of proficiency in determining what a good language learner is. (The Good Language Learner, L 39-41)

8- Wenden (1986) confirms that reading and discussing the strategies of good language learner is a constructive preliminary activity that helps students to get aware of the concept of learner's strategies. (Classification of Language Learning Strategies, $\mathbf{L}$ 52-54)

9- Most of the attempts to categorize language learning strategies definitely resulted into the same categorization of language learning strategies. (Classification of Language Learning Strategies, L 60-61)

10- Researchers are sure that degree of awareness, age, sex, nationality, among others, are factors that affect the choice of language learning strategies. (Factors Influencing the Choice of Learning Strategies, L 70-71)

11- Ehrman and Oxford (1989) concluded that women prefer social communication strategies more than men. (Factors Influencing the Choice of learning Strategies, L76-77).

12- After being exposed to more strategy training it is probable that men will use as much strategies as women. (Factors Influencing the Choice of learning Strategies , L77).

13-The findings have clearly shown that proficient students use more language strategies than other students. (Factors Influencing the Choice of Learning Strategies, L 78-80)

14- Research cannot yet tell us whether motivation affects the choice of learning strategies. (Factors Influencing the Choice of Learning Strategies, L 83-84)

15- Correlation between proficiency and strategy use is still to be verified. (Language Learning Strategies and Language Learning Achievement, L 110-111) 
B- Choose the best answer from the choices given:

16- From the section The Good Language Learner in \$1, we know for certain that:

a- that good learners are successful despite the teaching method adopted in class.

b- no single method and research finding would mark the start of universal success in teaching a second language.

c- some learners demonstrated that they lacked the abilities to succeed.

17- From the section Factors Influencing the Choice of Learning Strategies in §2, results of studies on gender concretely reveal that:

a- that after strategies training men and women will use the same strategies at the same frequency.

b- that women use strategies more often than men and with a greater variety.

c- that women prefer social communication strategies more than men.

18- From the section Factors Influencing the Choice of Learning Strategies in $\$ 3$ which statement is a conclusive finding that language learner styles play a crucial role in his choice of language learning strategies (§3):

a- Learning styles and learning strategies of an individual learner can work cooperatively with a given instructional method.

b- When harmony exists between these factors, the learner may perform well, feel confident and experience low anxiety.

c- Extroverts have demonstrated strong preference for social strategies while introverts use metacognitive strategies.

19- From the section Language Learning Strategies and Language Learning Achievement in \$1, we know for sure that: a- the acquisition of language learning strategies helps the learner to learn the target language more proficiently and facilitates the acquisition.

b- advanced language learners use learning strategies as frequently as elementary level students

c- once students learn to use learning strategies, they can maintain their motivation, autonomy and confidence and try to accomplish their goal of learning the target language

\section{Questionnaire :}

\section{Appendix C}

Each of the following sentences comes from the adapted reading text: Language Learning Strategies among EFL/ESL Learners: a Review of Literature. Please indicate how certain you think the writer expressed each of the statements below. Please mark each statement according to whether the writer wants to show she is "completely certain" (C), "fairly sure" (F), or "uncertain" (U), about the results or claim in. You can just respond with "I don't know (D)" if you cannot decide.

1 - Research findings in the area of second language acquisition have repeatedly verified the significant role that learners can play in the process of language learning. (Introduction, L2)

2-Teachers and researchers concluded that no single method of language teaching and research findings would mark the start of universal success in teaching a second language. (The Good Language Learner, L12-14)

3- It was realized that certain learners seemed to be successful regardless of methods on teaching techniques (The Good Language Learner L14-15)

4-Observations and research studies led researchers to describe "good" language learners in terms of personal characteristics, styles and strategies. (The Good Language Learner L16-17)

5- While the results of the studies on defining the good language learner are not based on empirical findings, they suggest characteristics of good language learners as students who are actively involved in language learning and are able to solve problems regarding their own learning. (The Good Language Learner L29-

30)

6- It has been consistently reported (Wenden and Rubin, 1987), that all language learners report or have been observed to using some types of strategies in learning a foreign or second language. (The Good Language Learner,L32-

34)

7- In addition, with this knowledge and awareness, students' language learning can be improved through learner training by their instructors. (The Good Language Learner, L 43-44)

8- In her study, Rubin (1975) argues that once identified, these strategies could be offered to less successful learners.

(Classification of Language Learning Strategies, L50-51)

9- For that reason it might not be appropriate to compare language learning strategies and assess their influence on the teaching and learning process. (Classification of Language Learning Strategies,L62-64)

10- But studying language learning strategies possibly will help both language teachers and language learners to understand language learning strategies and different methods which are involved in strategy use. (Classification of Language Learning Strategies, L64-65)

11 - By referring to the literature it seems that the most inclusive taxonomy of language learning strategies is provided by Oxford (1990). (Classification of Language Learning Strategies, L66-67)

12- The results of previous research studies have demonstrated that many factors affect the choice of learning strategies. (Factors Influencing the Choice of Learning Strategies, L 70-71)

13- Studies have shown significant gender differences between males and females in which females have demonstrated to use more and wider range of strategies than males. (Factors Influencing the Choice of Learning Strategies, L7475) 
14- These findings also strengthen the fact that strategy use is a complex phenomenon that interacts with a number of variables. (Factors Influencing the Choice of Learning Strategies, L 97-98)

15- O'Malley et al., (1985) clearly highlighted the importance of learning strategies by defining them as "any set of operations or steps used by a learner that will facilitate the acquisition, storage, retrieval or use of information" (p.23). (Language Learning Strategies and Language Learning Achievement, L 104-106). 